\title{
Editorial
}

\section{Mechanics and Geometry of Solids and Surfaces}

\author{
J. D. Clayton, ${ }^{1,2}$ M. A. Grinfeld, ${ }^{1}$ T. Hasebe, ${ }^{3}$ and J. R. Mayeur ${ }^{4}$ \\ ${ }^{1}$ Impact Physics, US ARL, Aberdeen, MD 21005-5066, USA \\ ${ }^{2}$ A. James Clark School (Adjunct), University of Maryland, College Park, MD 20742, USA \\ ${ }^{3}$ Department of Mechanical Engineering, Kobe University, Kobe 657-8501, Japan \\ ${ }^{4}$ Theoretical Division, Los Alamos National Laboratory, Los Alamos, NM 87545, USA
}

Correspondence should be addressed to J. D. Clayton; john.d.clayton1.civ@mail.mil

Received 5 June 2015; Accepted 2 July 2015

Copyright (C) 2015 J. D. Clayton et al. This is an open access article distributed under the Creative Commons Attribution License, which permits unrestricted use, distribution, and reproduction in any medium, provided the original work is properly cited.

\section{Introduction}

Invited were overview and original research papers on topics associated with mechanics and geometry of solids and surfaces. Contributors have diverse backgrounds in a number of technical disciplines, including theoretical and mathematical physics, pure and applied mathematics, engineering mechanics, or materials science. Submissions originating from North America, Europe, and Asia were received and peer-reviewed over a period of approximately one calendar year, spanning June 2014-June 2015. Invited research topics included but were not limited to the following: continuum physics and mechanics of materials, including nonlinear elasticity, plasticity, and higher-order gradient or micropolar theory [1]; mechanics and thermodynamics of moving surfaces [2], including phase transition fronts and shock waves; materials physics of crystal lattices, glasses, and interfaces in heterogeneous solids; multiphysics [3] and multiscale modeling; differential-geometric descriptions as applied to condensed matter physics and nonlinear science [4]; theory and new analytical solutions or new applications of existing solutions to related problems in mechanics, physics, and geometry; new developments in numerical methods of solution towards mechanics problems; and new physical experiments supporting or suggesting new theoretical descriptions. Published papers are grouped into four categories in what follows, wherein the content and relevance of each contribution are summarized. These categories are kinematics/geometry of surfaces (Section 2), electrostatics (Section 3), solid mechanics (Section 4), and thermal-fluid mechanics (Section 5).

\section{Kinematics/Geometry of Surfaces}

In "The Relationship between Focal Surfaces and Surfaces at a Constant Distance from the Edge of Regression on a Surface," the coauthors S. Yurttancikmaz and O. Tarakci investigate the relationship between focal surfaces and surfaces at a constant distance from the edge of regression on a surface. They show how focal surfaces of a manifold can be obtained by means of some special surfaces at a constant distance from the edge of regression on the manifold. Focal surfaces are known in the topic of line congruence which has been introduced in the general field of visualization. Applications include visualization of the pressure and heat distributions on an airplane and studies of temperature, rainfall, or ozone over the earth's surface. Focal surfaces are also used as an interrogation tool to analyze the quality of various structures before further processing in industrial settings, for example, in numerical controlled milling operations.

In "Weyl-Euler-Lagrange Equations of Motion on Flat Manifold," the author Z. Kasap studies Weyl-Euler-Lagrange equations of motion in a flat space. It is well known that a Riemannian manifold is flat if its curvature is everywhere zero. Furthermore, a flat manifold is one Euclidean space in terms of distances. Weyl introduced a metric with a conformal transformation for unified theory in 1918. Classical mechanics problems are often analyzed via the Euler-Lagrange equations. In this study, partial differential equations are obtained for movement of objects in space, and solutions of these equations are generated using symbolic algebra software. The present set of Euler-Lagrange mechanical equations derived on a generalization of flat manifolds may be suggested to deal 
with problems in electrical, magnetic, and gravitational fields, for the paths of defined space-moving objects.

In "The Steiner Formula and the Polar Moment of Inertia for the Closed Planar Homothetic Motions in Complex Plane," the coauthors A. Tutar and O. Sener express the Steiner area formula and the polar moment of inertia during one-parameter closed planar homothetic motions in the complex plane. The Steiner point or Steiner normal concepts are described according to whether a rotation number is different from zero or equal to zero, respectively. The moving pole point is given with its components, and its relation between a Steiner point and a Steiner normal is specified. The sagittal motion of a winch is considered as an example. This motion is described by a double hinge consisting of the fixed control panel of the winch and its moving arm. The winch is studied here because its arm can extend or retract during one-parameter closed planar homothetic motions.

\section{Electrostatics}

In "A Variational Approach to Electrostatics of Polarizable Heterogeneous Substances," the coauthors M. Grinfeld and P. Grinfeld discuss equilibrium conditions for heterogeneous substances subject to electrostatic or magnetostatic effects. The goal of this paper is to present a logically consistent extension of the Gibbs variational approach [2] to elastic bodies with interfaces in the presence of electromagnetic effects. It is demonstrated that the force-like aleph tensor and the energy-like beth tensor for polarizable deformable substances are divergence-free. Two additional tensors are introduced: the divergence-free energy-like gimel tensor for rigid dielectrics and the general electrostatic gamma tensor which is not necessarily divergence-free. The present approach is based on a logically consistent extension of the Gibbs energy principle that takes into account polarization effects.

Contrary to many prior attempts, explicitly excluded are the electric field and the electric displacement from the list of independent thermodynamic variables. Instead, polarization is treated by adding a single term to the traditional free energy for a thermoelastic system. The additional term represents the potential energy accumulated in the electrostatic field over the entire space. The exact nonlinear theory of continuous media is invoked with Eulerian coordinates as the independent spatial variables.

While the proposed model is mathematically rigorous, the authors caution against the assumption that it can reliably predict physical phenomena. On the contrary, clear models often lead to conclusions at odds with experiment and therefore should be treated as physical paradoxes that deserve the attention of the scientific community.

\section{Solid Mechanics}

In "On Finsler Geometry and Applications in Mechanics: Review and New Perspectives," the author J. D. Clayton begins with a review of necessary mathematical definitions and derivations and then reviews prior work involving application of Finsler geometry in continuum mechanics of solids. The use of Finsler geometry (e.g., [5]) to describe continuum mechanical behavior of solids was suggested nearly five decades ago by Kröner in 1968 [1]. As overlooked in the initial review by the author, Finsler geometry was applied towards deforming ferromagnetic crystals by Amari in 1962 [3] and has somewhat recently been applied to fracture mechanics problems [6]. Building on theoretical work of Ikeda [7], Bejancu [8] distinguished among horizontal and vertical distributions of the fiber bundle of a finitedeforming pseudo-Finslerian total space. More complete theories incorporating a Lagrangian functional (leading to physical balance or conservation laws) and couched in terms of Finsler geometry were developed by Stumpf and Saczuk for describing inelasticity mechanisms such as plasticity and damage [9], including the only known published solutions of boundary value problems incorporating such sophistication.

This contributed paper by J. D. Clayton also introduces aspects of a new theoretical description of mechanics of continua with microstructure. This original theory, though neither complete nor fully explored, combines ideas from finite deformation kinematics [10], Finsler geometry [5, 8], and phase field theories of materials physics. Future work will enable encapsulation of phase field modeling of fracture and possible electromechanical coupling within Finsler geometric framework.

\section{Thermal-Fluid Mechanics}

In "Comparison of Optimal Homotopy Asymptotic and Adomian Decomposition Methods for a Thin Film Flow of a Third Grade Fluid on a Moving Belt," the coauthors F. Mabood and N. Pochai investigate a thin film flow of a third-grade fluid on a moving belt using a powerful and relatively new approximate analytical technique known as the Optimal Homotopy Asymptotic Method (OHAM). Due to model complexities, difficulties often arise in obtaining solutions of governing nonlinear differential equations for non-Newtonian fluids. A second-grade fluid is one of the most acceptable fluids in this class because of its mathematical simplicity in comparison to third-grade and fourth-grade fluids. In related literature, many authors have effectively treated the complicated nonlinear equations governing the flow of a third-grade fluid. In this study, it is observed that the OHAM is a powerful approximate analytical tool that is simple and straightforward and does not require the existence of any small or large parameter as does the traditional perturbation method. The variation of the velocity profile for different parameters is compared with numerical values obtained by the Runge-Kutta-Fehlberg fourth-fifthorder method and with the Adomian Decomposition Method $(\mathrm{ADM})$. An interesting result of the analysis is that the threeterm OHAM solution is more accurate than five-term ADM solution, confirming feasibility of the former method.

In "Optimal Homotopy Asymptotic Solution for Exothermic Reactions Model with Constant Heat Source in a Porous Medium," the coauthors F. Mabood and N. Pochai consider analytical and numerical treatments of heat transfer in particular problems. Heat flow patterns/profiles are required for heat transfer simulation in various types of thermal 
insulation. The exothermic reaction models for porous media can often be prescribed in the form of sets of nonlinear ordinary differential equations. In this research, the driving force model due to temperature gradients is considered. A governing equation of the model is restructured into an energy balance equation that provides the temperature profile in a conduction state with a constant heat source in the steady state. A proposed Optimal Homotopy Asymptotic Method (OHAM) is used to compute the solutions of the exothermic reactions equations. The posited OHAM scheme is convenient to implement, has fourth-order accuracy, and demonstrates no obvious problematic instabilities.

$$
\begin{array}{r}
\text { J. D. Clayton } \\
\text { M. A. Grinfeld } \\
\text { T. Hasebe } \\
\text { J. R. Mayeur }
\end{array}
$$

\section{References}

[1] E. Kröner, "Interrelations between various branches of continuum mechanics," in Mechanics of Generalized Continua, E. Kröner, Ed., pp. 330-340, Springer, Berlin, Germany, 1968.

[2] M. A. Grinfeld, Thermodynamic Methods in the Theory of Heterogeneous Systems, Longman, Sussex, UK, 1991.

[3] S. Amari, "A theory of deformations and stresses of ferromagnetic substances by Finsler geometry," in RAAG Memoirs, K. Kondo, Ed., vol. 3, pp. 257-278, 1962.

[4] J. D. Clayton, Nonlinear Mechanics of Crystals, Springer, Dordrecht, The Netherlands, 2011.

[5] H. Rund, The Differential Geometry of Finsler Spaces, Springer, Berlin, Germany, 1959.

[6] I. A. Miklashevich, "Geometric characteristics of fractureassociated space and crack propagation in a material," Journal of Applied Mechanics and Technical Physics, vol. 44, no. 2, pp. 255-261, 2003.

[7] S. Ikeda, "A physico-geometrical consideration on the theory of directors in the continuum mechanics of oriented media," Tensor. New Series, vol. 27, pp. 361-368, 1973.

[8] A. Bejancu, Finsler Geometry and Applications, Ellis Horwood, New York, NY, USA, 1990.

[9] H. Stumpf and J. Saczuk, "A generalized model of oriented continuum with defects," Zeitschrift für Angewandte Mathematik und Mechanik, vol. 80, no. 3, pp. 147-169, 2000.

[10] J. D. Clayton, Differential Geometry and Kinematics of Continua, World Scientific, Singapore, 2014. 


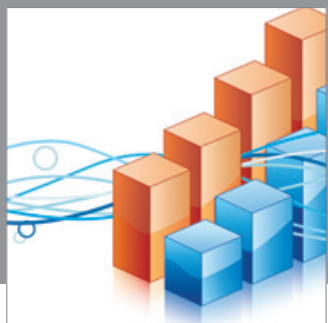

Advances in

Operations Research

mansans

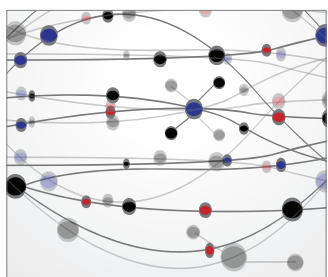

The Scientific World Journal
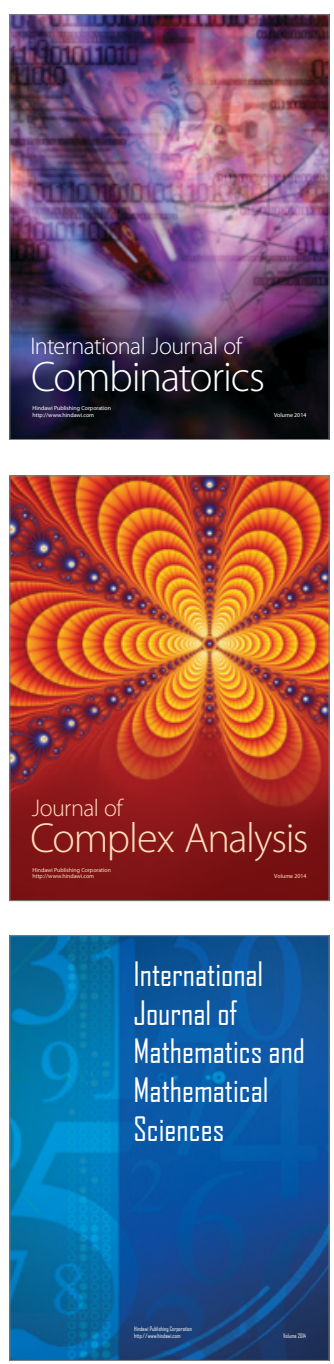
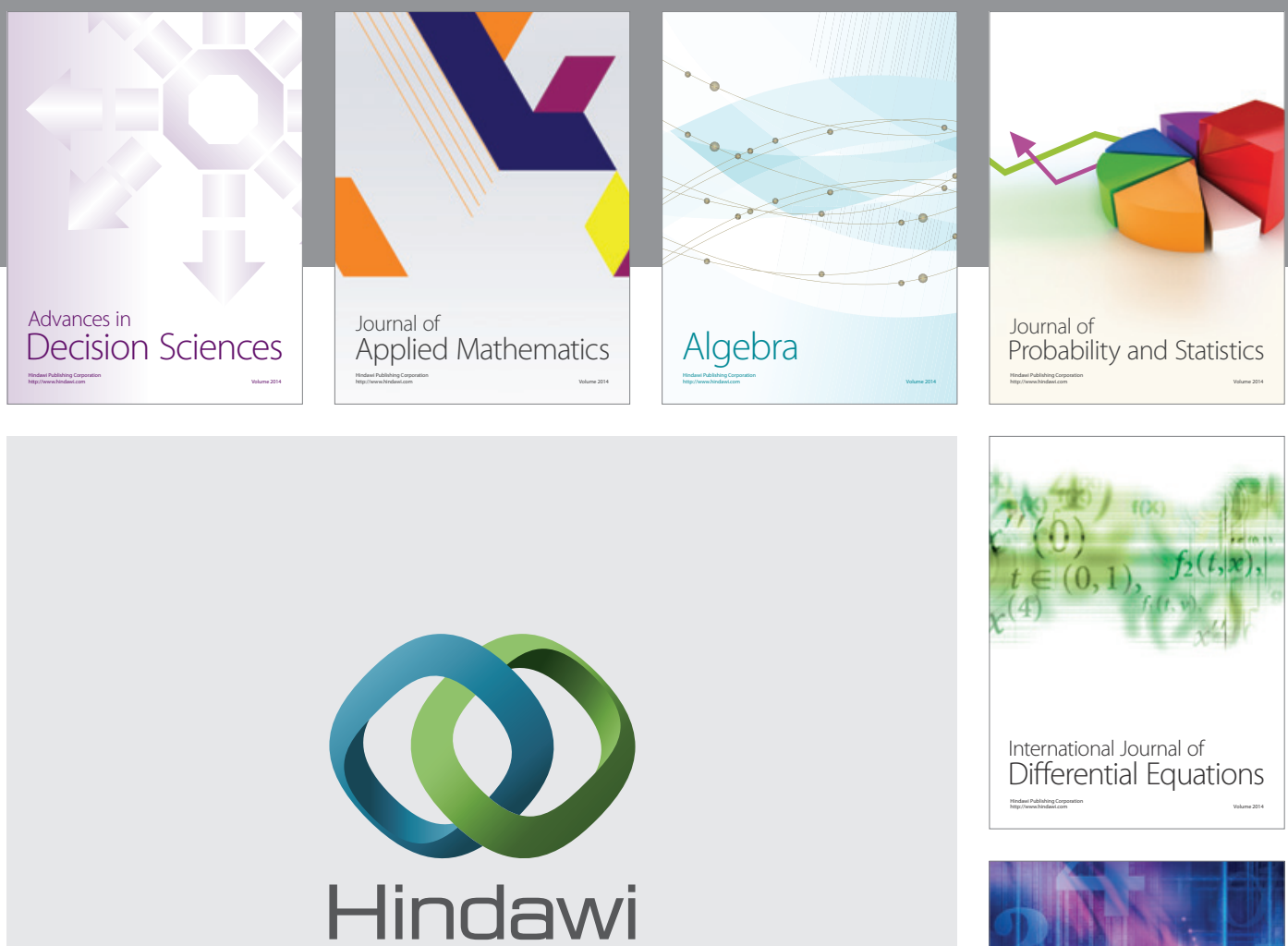

Submit your manuscripts at http://www.hindawi.com
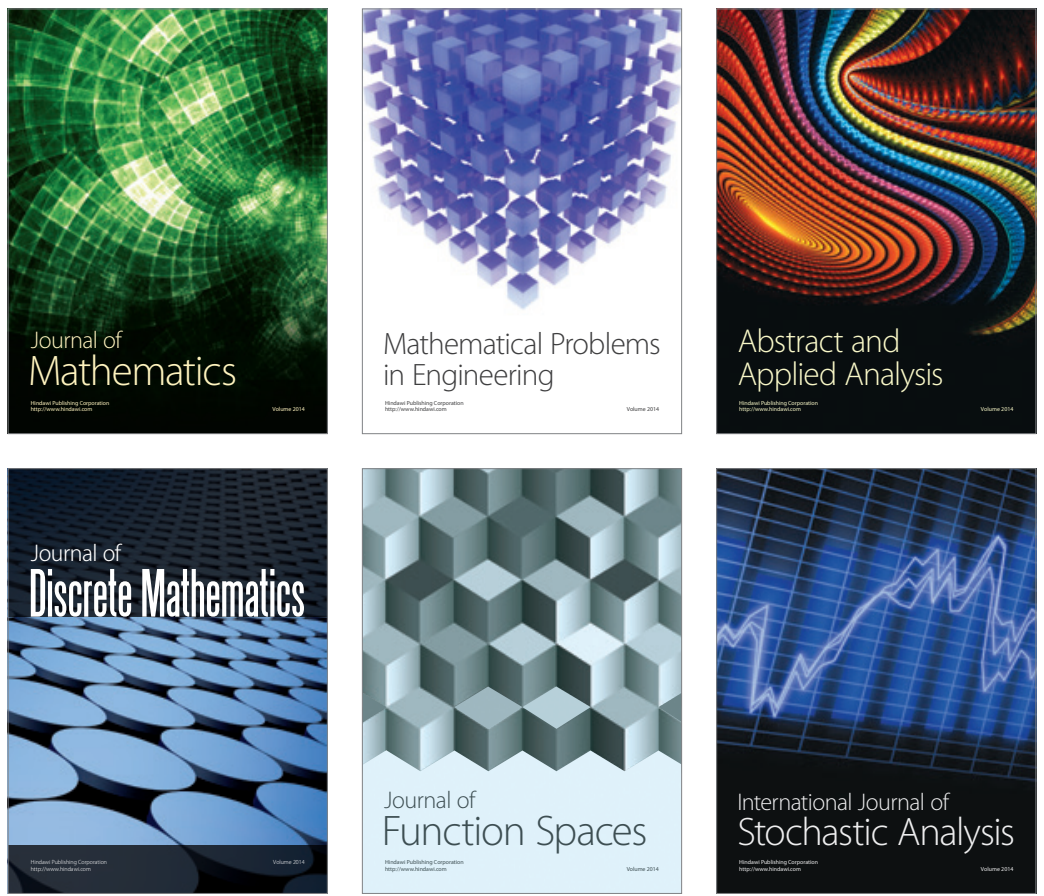

Journal of

Function Spaces

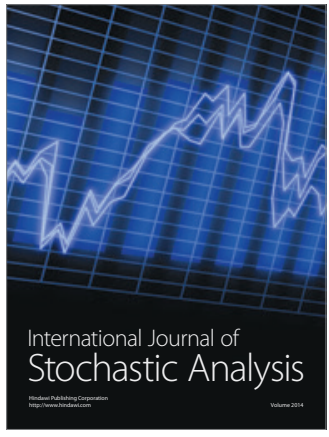

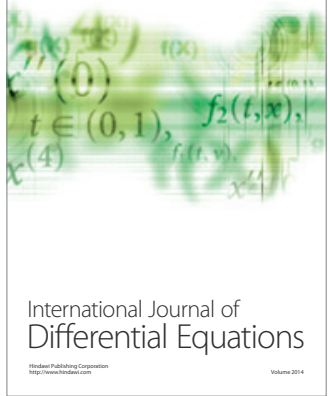
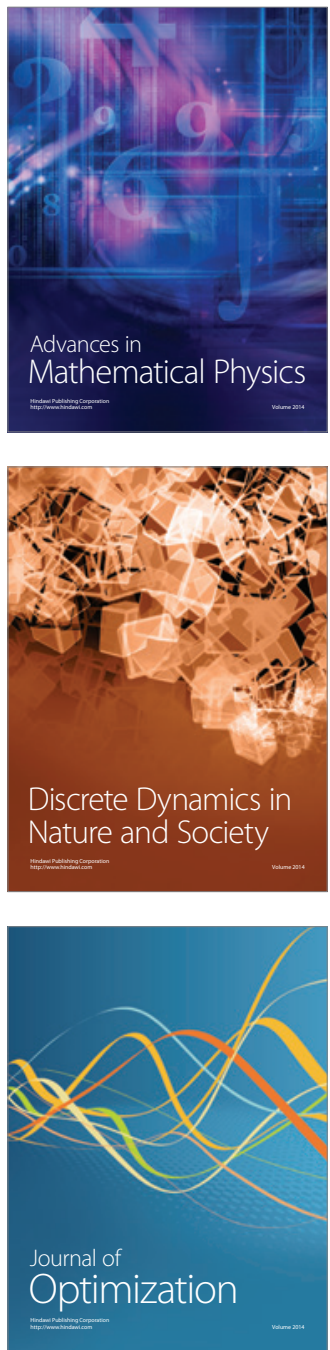